Pacific Journal of Mathematic 


\title{
ON THE HOMOMORPHISMS OF AN ALGEBRA ONTO FROBENIUS ALGEBRAS
}

\author{
W. E. DESKINS
}

1. Introduction. A linear associative algebra possessing a nonsingular parastrophic matrix is known as a Frobenius algebra after the mathematician who first investigated the properties of such an algebra [3]. In more recent years the properties of this class of algebras have been studied in papers by a number of mathematicians, notably R. Brauer, C. C. MacDuffee, T. Nakayama, and C. Nesbitt (see References).

Since Frobenius algebras are defined in terms of the parastrophic matrices, a natural question to ask is the following: Does a parastrophic matrix of rank $m$ of an algebra $C$ of order $n$ determine in some manner a homomorphism of $C$ onto a Frobenius algebra of order $m$ ? As the answer to this query is, in general, negative, it is the purpose of this paper to investigate the question: When does a parastrophic matrix of rank $m$ determine in some manner a homomorphism of $C$ onto a Frobenius algebra of order $m$ ? First a "manner of determination" is selected. Since the parastrophic matrices of $a$ form a double $a_{\text {-module, various }}$ ideals of $G$ of annihilating elements correspond to each parastrophic matrix. These are studied and conditions are developed (Theorem 9) which insure the determination from these annihilators an ideal $B$ such that the difference algebra $a-B$ is a Frobenius algebra of order $m$. These requirements are shown to be necessary, also, in the sense that any homomorphism of $C$ onto a Frobenius algebra of order $m$ implies the existence of a parastrophic matrix $Q$ of rank $m$ which satisfies these conditions. Furthermore, the kernel of the homomorphism will be the ideal $B$ determined from among those elements which annihilate $Q$ as an element of a double $C_{\text {-module. }}$

Basic terminology is introduced in $\$ 2$, parastrophic modules are defined, and the order of such a module is discussed. In $\$ 3$ one-sided ideals determined by the parastrophic matrices are considered, while $\$ 4$ is devoted to a study of two-sided ideals determined by certain parastrophic matrices and of the homomorphisms of an algebra onto Frobenius algebras. Certain of the ideals

Received February 10, 1954. Presented to the American Mathematical Society May $1,1954$.

Pacific J. Math. 5 (1955), 501-511 
introduced in $\$ 4$ have radical-like properties, and these ideals are considered in $\S 5$. A supplementary re $\quad \mathrm{s}$ on the order of the radical of a Frobenius algebra is given in $\$ 6$.

The author wishes to express his gratitude to Professor C. C. MacDuffee for his counsel during the preparation of this paper.

2. Preliminary remarks. Let $a$ be a linear associative algebra of order $n$ over the field $\mathcal{F}$, and let $e_{1}, \ldots, e_{n}$ be an $\mathcal{F}$-basis for $G$. Multiplication in $a$ follows from the multiplication of the basis elements,

$$
e_{i} e_{j}=\sum_{k} c_{i j k} e_{k} \quad \quad i, j=1, \cdots, n,
$$

where the $c_{i j k}$ are elements of $\mathcal{F}$, the constants of multiplication.

The associativity condition, written in terms of these constants of multiplication, is equivalent to each of the following sets of $n^{2}$ matric equations:

$$
\begin{array}{rlr}
Q_{j} R_{i} & =\sum_{k} c_{i k j} Q_{k}, & \\
S_{i} Q_{j} & =\sum_{k} c_{k i j} Q_{k} & i, j=1, \cdots, n,
\end{array}
$$

where the matrices $R_{i}, S_{i}$, and $Q_{i}$ are defined as $\left(c_{i s r}\right),\left(c_{r i s}\right)$ and $\left(c_{s r i}\right)$, respectively, where $r$ denotes the row and $s$ the column index.

Let $a \in G$; then

$$
a=a_{1} e_{1}+\cdots+a_{n} e_{n},
$$

where the $a_{i}$ are field elements. Let

$$
\begin{aligned}
& R(a)=a_{1} R_{1}+\cdots+a_{n} R_{n}, \\
& S(a)=a_{1} S_{1}+\cdots+a_{n} S_{n}, \\
& Q(a)=a_{1} Q_{1}+\cdots+a_{n} Q_{n} .
\end{aligned}
$$

$R(a)$ is called the first matrix, $S(a)$ the second matrix, and $Q(a)$ the parastrophic matrix, of $a$. (Note that $Q(a)$ as defined here is the transpose of the parastrophic matrix as defined by other authors). The set $R(\&)$ of all the first (second) matrices of $C_{\text {form }}$ an algebra which is a homomorphic image of $C_{\text {. The }}$ 
set 2 of all the parastrophic matrices of $C$ does not in general have this property, but if the following definitions are made,

$$
e_{i} * Q_{j}=S_{i} Q_{j}, Q_{j} * e_{i}=Q_{j} R_{i} \quad i, j=1, \cdots, n,
$$

then 2 is a double $G$-module, the parastrophic module of $C_{\text {. }}$.

If a change of basis is made for $G$, the elements of $R$ and \& undergo similarity transformations, while the elements of 2 undergo congruency transformations [8]. Hence rank and symmetry are invariant set properties of 2 .

MacDuffee has obtained [7] necessary and sufficient conditions that $R$ and \& be algebras isomorphic with $G$. A corresponding result for 2 is given by

THEOREM 1. 2 is of order $m$ (as an $(\mathrm{l}$-module) if and only if the following conditions are satisfied:

(i) A contains an ideal W of order $m$ such that the difference algebra a - W is a zero algebra.

(ii) A contains no ideal of lower order with this property.

The proof of this theorem is a standard reversible procedure involving a change of basis for $G$. Let there be $n-m$ linearly independent linear relations among the $Q_{i}$; then there exist $n-m$ linearly independent row vectors

$$
T_{i}=\left(t_{i 1}, \cdots, t_{i n}\right) \quad i=m+1, \cdots, n \text {, }
$$

such that

$$
\sum_{k} t_{i k} Q_{k}=0
$$

If $B$ is a nonsingular $n$ by $n$ matrix with the $T_{i}$ as its last $n-m$ rows, and if $u_{1}, \cdots, u_{n}$ form a new basis for $G$,

$$
u_{i}=\sum_{k} p_{k i} e_{k} \quad i=1, \cdots, n \text {, }
$$

where $P=\left(p_{r S}\right)=B^{-1}$, then the $Q_{i}$ are transformed into

$$
\begin{array}{rlrl}
Q_{i}^{\prime} & =P^{T}\left(\sum_{k} t_{i k} Q_{k}\right) P \\
& =0 & i>m .
\end{array}
$$


Thus if the new constants of multiplication are $c_{i j k}^{\prime}$, then

$$
c_{i j k}^{\prime}=0
$$

$i, j=1, \cdots, n ; k>m$

and $u_{1}, \cdots, u_{m}$ span an ideal $W$ of order $m$ such that $G$ - W is a zero algebra. The process is clearly reversible.

COROLLARY. If C has either a left or right identity element, then 2 is of order $n$.

$a$ is said to be a Frobenius algebra if 2 contains a nonsingular element.

THEOREM 2. Q is a Frobenius algebra if and only if 2 is a cyclic module of order $n$.

If 2 contains a nonsingular element $Q$, then (1) and (2) imply that

$$
a * Q=Q * a=2,
$$

and 2 is of order $n$ since a Frobenius algebra possesses an identity element. Conversely, if 2 is generated by an element $Q$ and is of order $n$, then (1), (2), and Theorem 1 imply that $Q$ is nonsingular.

3. Ideals of $C$. Let $B$ be a right ideal of $C$ of order $n-m$. If a basis is selected for $C$ such that the last $n-m$ elements of the basis span $B$, then the $m$ matrices $Q_{1}, \ldots, Q_{m}$ have all zeros in their last $n-m$ columns. The task of determining a right ideal by a process involving reduction of certain elements of 2 through changes of bases of $C$ seems formidable, if possible. However, a somewhat similar process is given by the following theorem.

THEOR EM 3. A parastrophic matrix $Q$ of rank $m$ determines a right ideal $B$ of order greater than or equal to $n-m$.

Let $B$ be the set of all elements $b \in Q$ such that $Q * b=0$. Clearly $B$ is a right ideal. That its order is at least $n-m$ will follow from the next theorem.

That $B$ may actually be of order greater than $n-m$ is proved by the following example. Let $G$ have basis elements $e_{1}$ and $e_{2}, e_{1}^{2}=e_{1} e_{2}=e_{2} e_{1}=0$, $e_{2}^{2}=e_{1}$. Then the corresponding $Q_{1}$ has rank 1 but $B=a_{\text {. }}$

A more desirable result is contained in the following.

THEOREM 4. A parastrophic matrix of rank $m$ determines a right ideal of order $n-m$. 
Let $Q$ be a parastrophic matrix of rank $m$. Then there exists a nonsingular $n$ by $n$ matrix $P$ such that the elements of the last $n-m$ columns of $Q P$ are all zeros. Let $P$ effect a change of basis for $A$; that is, if $P=\left(p_{r s}\right)$, let

$$
u_{i}=\sum_{k} p_{k i} e_{k} \quad i=1, \cdots, n
$$

be a new basis for $\mathrm{C}_{\text {. If }} Q=Q(a)$, then $Q^{\prime}(a)$, with respect to the new basis, is $P^{T} Q P$ and hence has nothing but zeros in its last $n-m$ columns.

Now assume $Q$ is of this form. Then

$$
Q=\sum_{i} a_{i} Q_{i}=\left(\sum_{i} a_{i} c_{s r i}\right)
$$

so that

$$
\sum_{i} a_{i} c_{j k i}=0
$$

From (1) and (3) it follows that

$$
Q R_{i}=\sum_{j} a_{j}\left(\sum_{k} c_{i k j} Q_{k}\right)=\sum_{k}\left(\sum_{j} a_{j} c_{i k j}\right) Q_{k}=0 \quad i>m .
$$

Hence $Q * e_{i}=0$ for $i>m$, and $B=\left(e_{m+1}, \cdots, e_{n}\right)$ is the right ideal determined by $Q$.

A right ideal of $a$ which may be determined in this way will be called a parastrophic right ideal.

THEOREM 5. A sufficient condition that the ideal of Theorem 3 be a parastrophic right ideal is that 2 be of order $n$.

Suppose $B=\left(e_{m+1}, \cdots, e_{n}\right)$ is determined from $Q$ as above, and consider $e_{i}, i \leq m$. If $Q * e_{i}=0$, then if 2 is of order $n,(4)$ implies

$$
\sum_{j} a_{j} c_{i k j}=0
$$$$
k=1, \ldots, n,
$$

which is impossible since $Q$ is assumed to be of rank $m$.

Let $Q=\sum a_{i} Q_{i}$ be in the reduced form described above. 
THEOREM 6. If $C$ has a right identity element, then $a_{i}=0$ for $i>m$.

Since $G$ has a right identity element there are field elements $f_{i}$ such that

$$
\sum_{i} f_{i} S_{i}=I, \quad \sum_{i} f_{i} c_{k i j}=\delta_{k j} .
$$

Then ( 3 ) implies

$$
\sum_{k} f_{k}\left(\sum_{i} a_{i} c_{j k i}\right)=0=\sum_{i} a_{i}\left(\sum_{k} f_{k} c_{j k i}\right)=\sum_{i} a_{i} \delta_{j i}=a_{j} \quad j>m .
$$

The results of this section are obviously valid if the word "right" is replaced by "left".

Since the existence of ideals in an algebra $C$ has been shown to be equivalent to the existence of singular elements in 2 , the following theorem is immediate.

THEOREM 7. $Q$ is a division algebra if and only if 2 contains no singular elements.

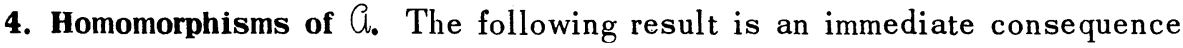
of Theorem 4 and its analogue for left ideals.

THEOREM 8. If $Q$ is congruent to a matrix of the form

$$
\left(\begin{array}{ll}
T & 0 \\
0 & 0
\end{array}\right)
$$

where $T$ is a nonsingular $m$ by $m$ matrix, then the right parastrophic ideal $B$ is also a left parastrophic ideal. Conversely, if $B$ is a right parastrophic ideal determined by $Q$, then if $B$ is also a two-sided ideal, $Q$ satisfies the above condition.

Such an ideal will be called a parastrophic ideal, and $Q$ will be said to have $P$-rank $m$. While $P$-rank is not defined for every matrix, it is a property of every symmetric matrix. Thus, if the characteristic of $\mathcal{F}$ is greater than $n$, the radical of $C$ is a parastrophic ideal. (It will be apparent shortly that this is true regardless of the field characteristic since a semisimple algebra is a Frobenius algebra. )

It does not follow that a matrix of 2 of $P$-rank $m$ determines a homomorphism 
of $C$ onto a Frobenius algebra of order $m$, for any commutative nilpotent nonzero algebra contains proper parastrophic ideals. The following indicates a necessary criterion.

LEMMA 1. If $\pi$ is a homomorphism of $\mathrm{C}$ onto $\mathrm{C}$, an algebra with an identity element 1 , then $Q$ contains an idempotent element $e$ such that $\pi e=1$. Furthermore, the set of left annihilators of $e$ is contained in the kernel of the homomorphism.

This follows simply from the structure theory for algebras.

Now suppose the last $n-m$ basis elements of $C$ form a parastrophic ideal $B$, and suppose that $C$ has an idempotent element $u$ such that $C_{u} \cup B=a_{\text {. }}$ Then $B$ will be called a regular parastrophic ideal.

THE OR Ем 9. A homomorphism of $\mathrm{G}$ onto a Frobenius algebra of order $m$ has as its kernel a regular parastrophic ideal of order $n-m$, and conversely if $B$ is a regular parastrophic ideal of $G$ of order $n-m$, then $G-B$ is a Frobenius algebra of order $m$.

Suppose $\bar{a}$ is a Frobenius image of $a$, with basis $\bar{e}_{1}, \ldots, \bar{e}_{m}$ and kernel $B$ spanned by $e_{m+1}, \ldots, e_{n}$.

Then $\bar{a}$ possesses a nonsingular $m$ by $m$ parastrophic matrix $\bar{Q}$,

$$
\bar{Q}=\sum_{i}^{m} a_{i} \bar{Q}_{i},
$$

and

$$
Q=\sum_{i}^{m} a_{i} Q_{i}
$$

is an element of 2 with $P$-rank $m$. Hence $B$ is a parastrophic ideal. By Lemma $1, B$ is a regular parastrophic ideal.

The converse follows from the regularity of $B$ and Theorem 6 .

Thus, if $Q$ is a parastrophic matrix of rank $m$, if $Q$ can be reduced to a corner matrix by a change of basis of $a$, and if $Q$ is associated with a linear combination of the first $m$ of the new basis elements, then $Q$ determines a homomorphism of $C$ onto a Frobenius algebra. Furthermore, each homomorphism of $C$ onto a Frobenius algebra may be determined in this fashion. 
5. Radical-like ideals. A function $f$ of $C$ into the set of all ideals of $C$ is called a radical function of $C$ if the contraction of $f$ to the difference algebra $C=a-f(a)$ maps $C$ onto the zero ideal. The ideal $f(a)$ is called a radicallike ideal of $\mathrm{a}$.

Let $\theta$ be the set of all regular parastrophic ideals of $C$ and let $n$ be an element of $P$ of minimal order, with the agreement that $n$ is the zero ideal if $a$ is a Frobenius algebra and $a$ if $C$ is nilpotent. Then define $f(a)=n$.

THEOREM 10. $f(a)=n$ is a nilpotent ideal of $a$.

If $a$ is nilpotent the theorem is trivially true, so assume that $a$ has the radical $\not \notin a$. Suppose $\not$ is of order $r$ and that $n$ is of order $m$. Let

$$
\ln \not{K}=C
$$

Case 1. $C=(0)$. Let $G$ have a basis such that the first $m$ basis elements span $\eta$ while the last $r$ span $\not{Z}$. By the definition of $\eta$ there is an element $Q^{\prime}$ of 2 of rank $n-m$ with its first $m$ rows and columns composed of only zeros. Now $n$ is isomorphic to a semisimple subalgebra of $G-\not{W}$, so there is an element $Q^{\prime \prime}$ of 2 of rank $m$ with only zeros in its last $n-m$ rows and columns. Then $Q^{\prime}+Q^{\prime \prime}$ is nonsingular.

Case 2. $C \neq(0)$. Then $C-C$ is a Frobenius algebra by the above work. In either case

$$
n \cap \not{\alpha}=n
$$

so that $\eta$ is contained in $\mathcal{K}$ and so is nilpotent.

One important property which $n$ may lack is uniqueness. The question of whether $h$ is unique up to an $G$-isomorphism will now be considered and partially answered.

The following result indicates a significance of the $C$-isomorphism of two minimal elements of $P$.

THEOREM 11. Let $\eta$ and $m$ be minimal elements of $P$. Then a necessary condition that $n$ and $m$ be $\mathrm{C}$-isomorphic is that $\mathrm{n}-\mathrm{C}$ and $\mathrm{m}-\mathrm{C}$ be zero algebras, where $\mathrm{C}=\mathrm{n}_{\mathrm{n}} \mathrm{m}$.

It may be assumed that $C=(0)$. Then let $\sigma$ be an $G$-isomorphism from $n$ onto m. If $a$ and $b$ are elements of $m$, then $n$ contains an element $b^{\prime}$ such that 


$$
a b=a\left(\sigma b^{\prime}\right)=\sigma\left(a b^{\prime}\right)=0 .
$$

The isomorphism of the minimal elements of $P$ for certain algebras will stem from the following lemma.

LEMMA 2. If $U$ and $V$ are $n$ by $n$ matrices of rank $m$ with elements from a field $\mathrm{F}$ which contains at least $m+1$ nonzero elements, then $\mathcal{F}$ contains an element $t \neq 0$ such that $U+t V$ is of rank at least $m$.

It will be sufficient to prove the result for $m=n$. Let $D$ and $E$ be nonsingular $n$ by $n$ matrices such that

$$
D V E=I \text {. }
$$

Consider the equation

$$
\operatorname{det}(D(U-x V) E)=\operatorname{det}(D U E-x I)=0,
$$

which is of degree $n$ in the indeterminate $x$. Since $r$ contains at least $n+1$ nonzero elements, one of them does not satisfy this equation.

Let $U$ and $V$ be $n$ by $n$ matrices, and let

$$
U \wedge V=0
$$

mean that the two matrices do not both have nonzero elements in the same rowcolumn position.

THEOREM 12. If two minimal elements $n_{1}$ and $n_{2}$ of $p$ are determined by symmetric matrices $Q_{1}$ and $Q_{2}$ of 2 of rank $m$, if $\mathcal{F}$ contains at least $m+1$ nonzero elements, and if

$$
Q_{1} \wedge Q_{2}=0
$$

then $a-n_{1}$ is isomorphic with $a-n_{2}$.

The cyclic modules

$$
a * Q_{1}=Q_{1} * a, \quad a * Q_{2}=Q_{2} * a
$$

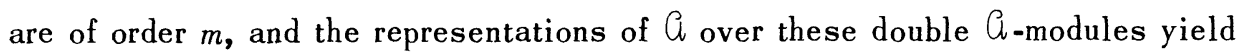
Frobenius algebras of order $m$ which are images of $a$, isomorphic with $a-n_{1}$ and $C-n_{2}$ respectively. Let $t$ be a nonzero element of $F$ such that $Q_{1}+t Q_{2}$ is of rank $m$ (since higher rank would contradict the minimality of the order of $n_{1}$ 
and $\left.n_{2}\right)$. Since $Q_{1}$ and $Q_{2}$ are symmetric

$$
a *\left(Q_{1}+t Q_{2}\right)=\left(Q_{1}+t Q_{2}\right) * a
$$

is a cyclic module of order $m$, and since $Q_{1} \wedge Q_{2}=0,(1)$ and (2) imply that the mapping

$$
\left(Q_{1}+t Q_{2}\right) R_{i} \rightarrow Q_{1} R_{i} \quad i=1, \cdots, n
$$

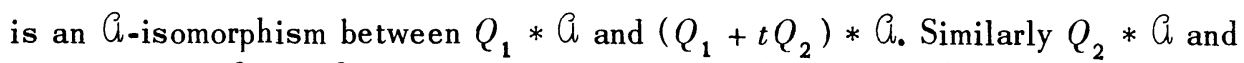

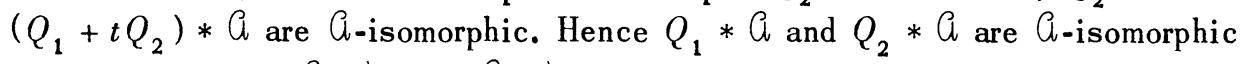
which implies that $a-n_{1}$ and $a-n_{2}$ are isomorphic.

6. A remark concerning Frobenius algebras. While Frobenius algebras are generally regarded as algebras with radicals of sufficiently small order, the following indicates that their radicals must also be of sufficiently large order.

THE OREM 13. Let $\mathrm{C}$ be a Frobenius algebra bound to its radical $\mathfrak{K}_{\text {. Then }}$

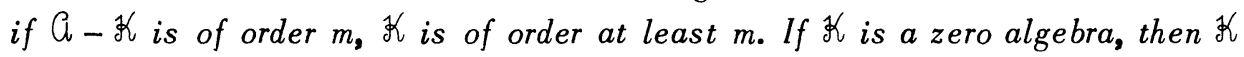
is of order $m$.

By the results of Nakayama [9] the set of all elements of $C$ which annihilate $\mathcal{K}$ from the right is an ideal $\mathcal{L}$ which also annihilates $\mathcal{Z}$ from the left and has order $n-k=m$, where $k$ is the order of $\mathcal{K}_{\text {. Since }} G$ is bound [4] to $\mathcal{K}$,

$$
\mathcal{L} \subseteq \mathscr{K}
$$

hence $m \leq k$, and $m=k$ if $\mathscr{L}=\mathcal{K}$.

The consideration of bound algebras is, of course, sufficient since an algebra may be written as a direct sum of a semisimple algebra and a bound algebra. (This result is due to M. Hall [4]).

\section{REFERENCES}

1. R. Brauer, On hyperkomplex arthmetic and a theorem of Speiser, A Speiser Festschrift, Zurich, 1945.

2. R. Brauer, and C. Nesbitt, On the regular representations of algebras, Proc. Nat. Acad. Sci., 23 (1937), $236-240$.

3. G. Frobenius, Theorie der hyperkomplexen Groessen, S.-B. Preuss. Akad. Wiss., Berlin (1903), 504- 537.

4. M. Hall, The position of the radical in an algebra, Trans. Amer. Math. Soc., 48 (1940), 391 - 404. 
5. M. Ikeda, and T. Nakayama, Supplementary remarks on Frobeniusean algebras, Osaka Math. J., 2 (1950), 7-12.

6. C. C. MacDuffee, Modules and ideals in a Frobenius algebra, Monatsh. Math. Phys., 48 (1939), $292-313$.

7. - On the independence of the first and second matrices of an algebra, Bull. Amer. Math. Soc., 35 (1929), 344-349.

8. , The theory of Matrices, Chelsea, New York, 1946.

9. T. Nakayama, On Frobeniusean algebras, I, Ann. Math., 40 (1939), 611-633.

10. - On Frobeniusean algebras, II, Ann. Math., 42 (1941), 1 - 21.

11. - On Frobeniusean algebras, III, Jap. J. Math., 18 (1942),

12. - Orthogonality relations for Frobenius and quasi-Frobenius algebras, Proc. Amer. Math. Soc., 3 (1952), 183 - 195.

13. C. Nesbitt, On the regular representations of algebras, Ann. Math, 39 (1938), $634-658$.

14. M. Osima, Some studies on Frobenius algebras, Jap. I. Math., 21 (1951), 179-190.

15. G. Simura, On a certain ideal of the center of a Frobenius ean algebra, Sci. Papers Coll. Gen. Ed. Univ. Tokyo, 2 (1952), $117-124$.

The OHIO State UnIVERSiTy 



\section{PACIFIC JOURNAL OF MATHEMATICS}

\section{EDITORS}

\section{H.L. ROY DEN}

Stanford University

Stanford, California

E. HEWITT

University of Washington

Seattle 5, Washington

\section{R. P. DILWORTH}

California Institute of Technology

Pasadena 4, California

* Alfred Horn

University of California

Los Angeles 24, California

\section{ASSOCIATE EDITORS}

\begin{abstract}
H. BUSEMANN
HERBERT FEDERER

MARSHALL HALL
\end{abstract}

\section{P.R. HALMOS}

HEINZ HOPF

ALFRED HORN
R.D. JAMES

BØRGE JESSEN

PAUL LÉVY
GEORGE PÓLYA

J.J. STOKER

KOSAKU YOSIDA
UNIVERSITY OF BRITISH COLUMBIA CALIFORNIA INSTITUTE OF TECHNOLOGY UNIVERSITY OF CALIFORNIA, BER KELEY UNIVERSITY OF CALIFORNIA, DAVIS UNIVERSITY OF CALIFORNIA, LOS ANGELES UNIVERSITY OF CALIFORNIA, SANTA BARBARA MONTANA STATE UNIVERSITY

UNIVERSITY OF NEVADA

OREGON STATE COLLEGE

UNIVERSITY OF OREGON
UNIVERSITY OF SOUTHERN CALIFORNIA STANFORD UNIVERSITY UNIVERSITY OF UTAH WASHINGTON STATE COLLEGE UNIVERSITY OF WASHINGTON

AMERICAN MATHEMATICAL SOCIETY HUGHES AIRCRAFT COMPANY SHELL DEVELOPMENT COMPANY

Mathematical papers intended for publication in the Pacific Journal of Mathematics should be typewritten (double spaced), and the author should keep a complete copy. Manuscripts may be sent to any of the editors. Manuscripts intended for the outgoing editors should be sent to their successors. All other communications to the editors should be addressed to the managing editor, Alfred Horn, at the University of California Los Angeles 24, California.

50 reprints of each article are furnished free of charge; additional copies may be obtained at cost in multiples of 50 .

The Pacific Journal of Mathematics is published quarterly, in March, June, September, and December. The price per volume (4 numbers) is $\$ 12.00$; single issues, $\$ 3.50$; back numbers (Volumes $1,2,3$ ) are available at $\$ 2.50$ per copy. Special price to individual faculty members of supporting institutions and to individual members of the American Mathematical Society: $\$ 4.00$ per volume; single issues, $\$ 1.25$.

Subscriptions, orders for back numbers, and changes of address should be sent to the publishers, University of California Press, Berkeley 4, California.

Printed at Ann Arbor, Michigan. Entered as second class matter at the Post Office, Berkeley, California.

* During the absence of E.G. Straus.

UNIVERSITY OF CALIFORNIA PRESS - BERKELEY AND LOS ANGELES 


\section{Pacific Journal of Mathematics}

\section{Vol. 5, No. $4 \quad$ December, 1955}

Richard Horace Battin, Note on the "Evaluation of an integral occurring in servomechanism theory" ............................. 481

Frank Herbert Brownell, III, An extension of Weyl's asymptotic law for eigenvalues................................. 483

Wilbur Eugene Deskins, On the homomorphisms of an algebra onto Frobenius algebras .................................. 501

James Michael Gardner Fell, The measure ring for a cube of arbitrary dimension ....................................... 513

Harley M. Flanders, The norm function of an algebraic field extension. II ............................................ 519

Dieter Gaier, On the change of index for summable series ............ 529

Marshall Hall and Lowell J. Paige, Complete mappings of finite groups . . . . 541

Moses Richardson, Relativization and extension of solutions of irreflexive relations..................................... 551

Peter Scherk, An inequality for sets of integers .................. 585

W. R. Scott, On infinite groups ........................... 589

A. Seidenberg, On homogeneous linear differential equations with arbitrary constant coefficients ......................................... 599

Victor Lenard Shapiro, Cantor-type uniqueness of multiple trigonometric integrals...

Leonard Tornheim, Minimal basis and inessential discriminant divisors for a cubic field...

Helmut Wielandt, On eigenvalues of sums of normal matrices ... 